\title{
LEHRE VON DEN GESICHTSEMPFINDUNGEN
}

AUF GRUND HINTERLASSENER AUFZEICHNUNGEN

VON

\section{FRANZ HILLEBRAND}

HERAUSGEGEBEN VON

Dr. FRANZISKA HILLEBRAND

MIT 40 TEXTABBILDUNGEN

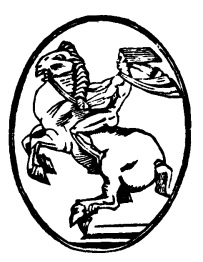

W I E N

VERLAG VON JULIUS SPRINGER

1929 
ALLE RECHTE, INSBESONDERE DAS DER ÜBERSETZUNG

IN FREMDE SPRACHEN, VORBEHALTEN

COPYRIGHT 1929 BY JULIUS SPRINGER IN VIENNA

ISBN-13: 978-3-7091-5655-1 e-ISBN-13: 978-3-7091-5693-3

DOI: 10.1007/978-3-7091-5693-3 


\section{Vorwort der Herausgeberin}

Meines Mannes experimentelle Arbeit war vorwiegend den Gesichtsempfindungen gewidmet. Er hat die Ergebnisse seiner Forschungen in einer Reihe von Publikationen niedergelegt, die chronologisch geordnet am Schlusse dieses Buches angeführt werden. Eine abschließende Zusammenfassung war seit langem beabsichtigt, doch wurde sie immer wieder hinausgeschoben, weil die Auseinandersetzung mit einzelnen, schwierigen Problemen wichtiger erschien. So sollte schließlich die Herausgabe einer Gesamtdarstellung der Lehre von den Gesichtsempfindungen - im Rahmen einer allgemeinen Psychologie - einer späteren Zeit vorbehalten bleiben, die auch die Befreiung von Vorlesungsverpflichtung und anderer amtlicher Tätigkeit gebracht hätte. Ein solcher, ruhiger Sammelarbeit gewidmeter Lebensabend war aber meinem Manne nicht beschieden, der Tod hatte ihn mitten aus seinen Arbeiten herausgerissen. Mir ist es vergönnt gewesen, in den letzten 10 Jahren seines Lebens zuerst seine Schülerin, dann seine Mitarbeiterin zu sein und als solche glaube ich die Verpflichtung zu haben, die Ergebnisse einer mehr als 30-jährigen rastlosen Forschertätigkeit, die nur zum kleineren Teile veröffentlicht wurden, nicht verloren gehen zu lassen. Die im Nachlasse vorgefundenen Darlegungen zu den Problemen der Ruhe und Bewegung gesehener Objekte wurden als „Kritischer Nachtrag zur Lehre von der Objektruhe bei willkürlichen Blickbewegungen und ihrer Anwendung auf die Stroboskopie" kürzlich in der Zeitschrift für Psychologie Bd. 104 u. 105 mitgeteilt. Es folgt nunmehr die Veröffentlichung der ,Lehre von den Gesichtsempfindungen“, die sich vorwiegend auf die sorgfältig ausgearbeiteten Vorlesungshefte, außerdem auf unveröffentlichte Vorträge, Notizen und dgl. stützt. An manchen Stellen schien es mir notwendig, auf frühere Publikationen meines Mannes und auf gegnerische Ansichten zu verweisen; diese Einschübe sind als „Anmerkungen der Herausgeberin“ gekennzeichnet.

Auf die neuere Literatur wurde in Fußnoten verwiesen. Von einem ausführlichen Literaturverzeichnis habe ich abgesehen; ein solches findet sich für den Raumsinn bei F. B. Hofmann, „Die Lehre vom Raumsinn des Auges“" (Handbuch der Augenheilkunde, Berlin, Julius Springer 1925). Für den Lichtsinn verweise ich auf die Zusammenstellung der älteren Literatur von A. KöNIG in der 2. Auflage des Handbuches der Physiologischen Optik von Hецмноцтz (1896), ferner auf die Literaturübersicht 
bei A. v. Tschermak, ,Kontrast und Irradiation“ (Ergebnisse der Physiologie 1903) und bei C. v. Hess, „Farbenlehre“ (Ergebnisse der Physiologie 1922), sowie auf die zur Zeitschrift für Psychologie und Physiologie der Sinnesorgane erscheinenden Registerbände.

Auf die grundlegenden Arbeiten E. HeRINGs, die von entscheidendem Einflusse auf die Anschauungen und auf die Methodik meines Mannes gewesen sind, wird immer wieder Bezug genommen. „Die Selbstzucht, die strenge Kritik, mit der der Forschende sich selbst wie einer fremden Person gegenüber tritt, die unbedingte Ehrlichkeit, die ihn treibt, Schwierigkeiten eher aufzusuchen, als über sie hinwegzugleiten" (HILlEBrand, „Ewald Hering“" S. 108), Eigenschaften, die jedem Forscher zukommen sollten und die bei Hering sich zu einer seltenen Vollkommenheit entwickelt hatten, wird man auch Hillebrand in einem das Gewöhnliche übersteigenden Maße zusprechen dürfen.

Innsbruck, im Sommer 1928

Dr. Franziska Hillebrand 


\title{
Inhaltsverzeichnis
}

\author{
Erster Teil
}

\section{Lichtsinn}

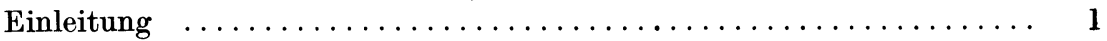

I. Die Abhängigkeit der Farbenempfindung vom Reiz ......... 3

1. Die drei Variablen der Farbenempfindungen: Farbenton, Hellig-

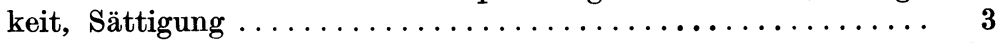

2. Methoden und Gesetze der Lichtmischung ........... 27

II. Die Abhängigkeit der Farbenempfindung von der Erregbarkeit . . 46

1. Adaptation und Kontrast ................... 46

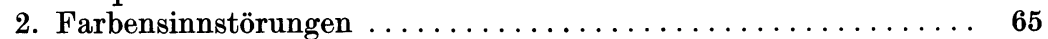

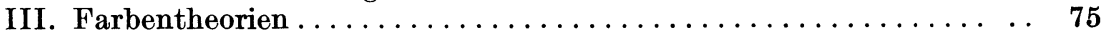

1. Entstehung und Aufgaben der Farbentheorien .......... 75

2. Die Young-Helmholtzsche Farbentheorie ............ 77

3. Die Theorie der Gegenfarben von E. Hering .......... 82

\section{Zweiter Teil}

\section{Raumsinn}

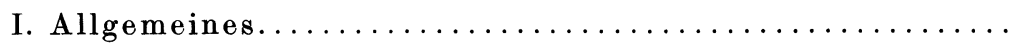
Wirklicher Raum und Sehraum. Bestimmte und unbestimmte, richtige und unrichtige Lokalisation. Ein paar Sätze aus der geometrischen Optik. Terminologische Festsetzungen.

II. Die Lokalisation bei ruhendem Blick ............ 103

A) Die ,primitive“ Raumanschauung ............... 103

1. Die Korrespondenz der Netzhäute und das Gesetz der iden-

tischen Sehrichtungen .................... 103

2. Der Horopter und die Kernfläche............... 114

3. Das Sehen mit disparaten Netzhautstellen .......... 121

4. Die Lokalisation des Kernpunktes .............. 143

B) Die empirischen Lokalisationsmotive .............. 150

III. Die Lokalisation bei bewegtem Blick ............ 155

1. Die Unveränderlichkeit der relativen Raumwerte ......... 156

2. Ruhe der Objekte bei willkürlichen, Scheinbewegung bei unwillkürlichen Blickbewegungen. ................ 156

3. Lokalisation bei Kopf- und Körperbewegungen .......... 167

IV. Nativismus und Empirismus .................. 169

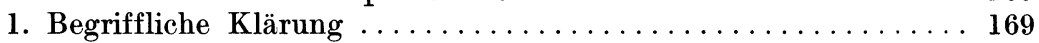

2. Die Argumente der Empiristen gegen den Nativismus . . . . 173

3. Kritik des Empirismus..................... 191

Verzeichnis der Publikationen Hillebrands aus dem Gebiete der

Lehre von den Gesichtsempfindungen ..............201

Sach verzeichnis . . . . . . . . . . . . . . . . . . 202 